\title{
Orientation of molecular interface dipole on metal surface investigated by noncontact atomic force microscopy
}

\author{
YUAN BingKai, CHEN PengCheng, ZHANG Jun, CHENG ZhiHai*, QIU XiaoHui* \& \\ WANG Chen*
}

National Center for Nanoscience and Technology, Beijing 100190, China

Received April 22, 2013; accepted June 5, 2013; published online July 12, 2013

\begin{abstract}
We investigated the orientations of interface dipole moments of individual non-planar titanyl phthalocyanine (TiOPc) molecules on $\mathrm{Cu}(111)$ and $\mathrm{Cu}(100)$ substrates using scanning tunneling microscope (STM) and noncontact atomic force microscope (NC-AFM). The dipole moment orientations corresponding to two different configurations of individual TiOPc molecules were determined unambiguously. The correlation between the actual molecular structures and the corresponding STM topographies is proposed based on the sub-molecular resolution imaging and local contact potential difference (LCPD) measurements. Comparing with the pristine substrate, the LCPD shift due to the adsorption of non-planar molecule is dependent on the permanent molecular dipole, the charge transfer between the surface and the molecule, and the molecular configurations. This work would shed light on tailoring interfacial electronic properties and controlling local physical properties via polar molecule adsorption.
\end{abstract}

titanyl phthalocyanine, interface dipole, non-contact atomic force microscopy, scanning tunneling microscopy, Kelvin probe force microscopy

Citation: Yuan B K, Chen P C, Zhang J, et al. Orientation of molecular interface dipole on metal surface investigated by noncontact atomic force microscopy. Chin Sci Bull, 2013, 58: 3630-3635, doi: 10.1007/s11434-013-5977-x

Interface dipoles induced by self-assembly of organic molecules on metal surfaces play an important role in controlling the surface and/or interface properties, especially in the modification of work function of the metal substrate and the energy-level alignment of organic semiconductor with the metal Fermi level [1,2]. Interface dipole is strongly influenced by the permanent dipole of the organic molecule and the "bonding" dipole induced by the molecule-metal interaction [3-6]. To understand the energy-level alignment at the interface and design interfaces with desired properties, atomic level investigations on the molecular structures of organic adsorbates and interfacial electronic structures are highly required and challenging.

Metal phthalocyanines (MPcs), together with their derivatives, have attracted great interest over the past years because of their unique optical and electrical properties [7]. Non-planar MPcs, such as titanyl phthalocyanine (TiOPc), tin phthalocyanine (SnPc) [8], vanadyl phthalocyanine (VOPc) $[9,10]$, chloroaluminum phthalocyanine (ClAlPc) [5] and chlorogallium phthalocyanine $(\mathrm{GaClPc})$ [3], may adsorb on metal surface with two opposite molecular orientations, which give rise to different dipole moments (Figure 1(b)). Different adsorption configurations have significant influence on the functionalities of molecules and surface and/or interface properties. However, the studies on the effects of the non-planar molecular structure and interfacial electronic structure on interface dipole are still limited. It is thus important to investigate the charge redistribution across the molecule and the metal substrate and to determine the orientations of interface dipole moments induced by different adsorbed configurations of non-planar molecules on metal surface.

The local contact potential difference (LCPD) [11] reflects the local character of the work function (WF), which

*Corresponding authors (email: chengzh@nanoctr.cn; xhqiu@nanoctr.cn; wangch@nanoctr.cn) 
is sensitive to microscopic structure of the surface. Interface dipole can shift the local WF of the sample in either direction and therefore, increase or decrease the LCPD. Electrostatic force measurements performed in noncontact atomic force microscope (NC-AFM) operation mode can be used to measure the LCPD between a conducting tip and the substrate surface at the atomic scale [12]. With the recent development of qPlus-AFM technique, the spatial resolution and energy sensitivity have reached single atom/molecule and single electron level $[13,14]$. For example, charge state of a single atom on thin insulating films and charge state of point defects of $\mathrm{MgO}$ surface have been discriminated $[13,15]$. Recently, Mohn et al. [14] demonstrated the mapping of the LCPD with sub-molecular resolution using Kelvin probe force microscopy (KPFM), and verified the reflection of the intra-molecular charge distribution by the LCPD mapping. Thus, measuring LCPD using NC-AFM could facilitate the unambiguous determination of the interface dipole moment orientation induced by adsorption of polar molecules on metal surface, which could reveal the charge transfer between the molecule and the metal surface at single molecule level.

In this paper, we investigate the interface dipoles corresponding to two different configurations of individual TiOPc molecules on $\mathrm{Cu}(111)$ and $\mathrm{Cu}(100)$ substrates by using NC-AFM based on a qPlus sensor design (qPlus NC-AFM) [16]. The interface dipole moments have the opposite orientations, which reflect the two different configurations of TiOPc molecules on $\mathrm{Cu}(111)$ surface. The actual molecular structures of the two configurations are correlated with the corresponding scanning tunneling microscope (STM) topographies by combining the sub-molecular resolved STM images and LCPD measurements. However, the interface dipole moments induced by the different configurations of TiOPc molecules on $\mathrm{Cu}(100)$ have the same orientation. This indicates that the TiOPc molecule in the O-down configuration interacts stronger with the $\mathrm{Cu}(100)$ surface than $\mathrm{Cu}(111)$ surface and large charge is donated from the $\mathrm{Cu}(100)$ substrate to the molecule. Compared with the pristine substrate, the LCPD shift due to the adsorbed nonplanar molecule is dependent on the permanent dipole moment of the molecule, the charge transfer between the substrate and the molecule, and the molecular configurations.

\section{Experimental}

The experiments were carried out with a commercial low temperature scanning probe microscope (Omicron Nanotechnology, Germany) operated at $\sim 78 \mathrm{~K}$ with a base pressure below $10^{-10} \operatorname{mbar}(1 \mathrm{mbar}=100 \mathrm{~Pa})$. The instrument is based on a qPlus sensor design, which can work at both STM and NC-AFM operation modes. The $\mathrm{Cu}(111)$ and $\mathrm{Cu}(100)$ surfaces were cleaned by cycles of $\mathrm{Ne}^{+}$sputtering $(1500 \mathrm{eV})$ followed by annealing at $\sim 850 \mathrm{~K}$. TiOPc mole- cules (97\% purity, Aldrich, USA) were in situ deposited from a heated homemade crucible on the $\mathrm{Cu}(111)$ and $\mathrm{Cu}(100)$ substrates on the STM stage at $\sim 78 \mathrm{~K}$. The molecules were degassed for more than $24 \mathrm{~h}$ before the deposition. An electrochemically etched tungsten tip was glued on the free prong of the tuning fork of the qPlus sensor. The resonant frequency of the qPlus sensor used for this study was $\sim 21 \mathrm{kHz}$ and the quality factor $\mathrm{Q}$ was in the range of 3000-6000. All voltages are referred to the sample bias with respect to the tip. During the LCPD measurements, a stable tip configuration is ensured and no tip condition change has been observed after the measurements.

\section{Results and discussion}

TiOPc is a typical non-planar phthalocyanine molecule with a significant vertical permanent dipole moment of 3.73 Debye [17], as schematically shown in Figure 1(a). In contrast to the planar MPcs, the Ti and $\mathrm{O}$ atoms of TiOPc are located above the molecular plane. The aromatic Pc prefers to adopt a flat-lying configuration when adsorbed onto metal or graphite surfaces as reported in previous works [18]. The molecular configurations of non-planar TiOPc on surface could be $\mathrm{O}$ atom pointing to the vacuum (O-up with dipole pointing to the substrate) and the substrate (O-down with dipole pointing to the vacuum) as shown in Figure 1(b).
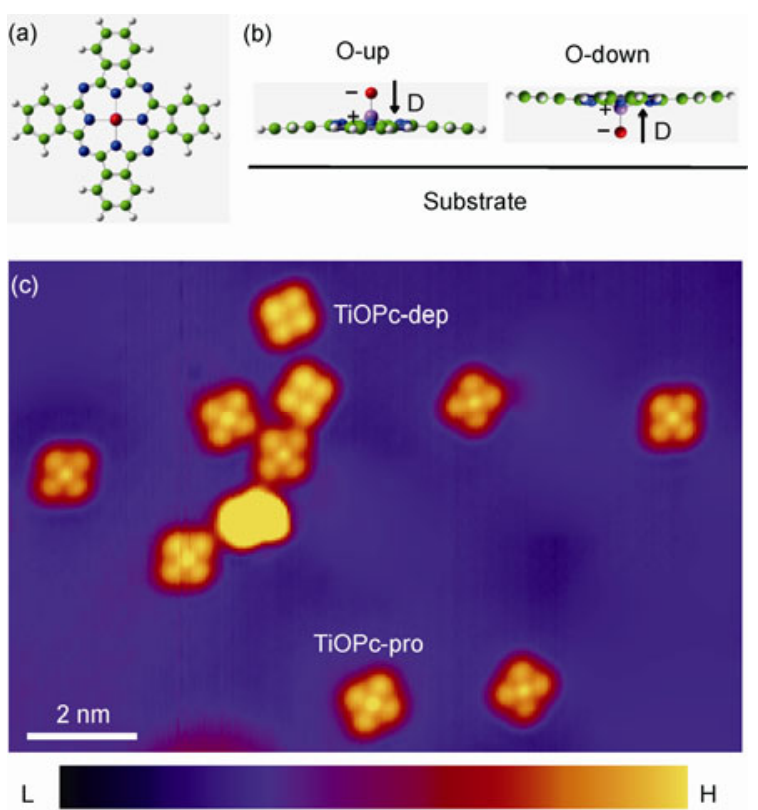

Figure 1 (a) Chemical structure of TiOPc molecule in top view with a vertical dipole moment of 3.73 Debye. The different atomic species are colored in purple $(\mathrm{Ti})$, red $(\mathrm{O})$, blue $(\mathrm{N})$, green $(\mathrm{C})$ and white $(\mathrm{H})$. (b) Schematic representation of two configurations of TiOPc on surface, O-up ( $\mathrm{O}$ atom pointing to the vacuum, dipole-down) and $\mathrm{O}$-down $(\mathrm{O}$ atom pointing to the substrate, dipole-up). (c) Constant-current STM image of TiOPc-dep and TiOPc-pro molecules on the $\mathrm{Cu}(111)$ surface $\left(I_{\mathrm{t}}=50 \mathrm{pA}\right.$, $\left.V_{\text {bias }}=1.0 \mathrm{~V}\right)$ 
Figure 1(c) shows a typical STM image of TiOPc molecules deposited on the $\mathrm{Cu}(111)$ surface. Two distinct configurations of the TiOPc molecules were clearly resolved. One type of molecular configuration exhibits a four-lobed "cross" structure with a central protrusion, referring to TiOPc-pro configuration. The other configuration presents a four-lobed structure with a central depression, referring to TiOPc-dep configuration. Different to the adsorption on $\operatorname{Ag}(111)$ surface at room temperature, on which TiOPc molecules adsorbed in a tilted configuration and exhibited a three-lobe shape in STM images [19,20], the four-lobed "cross" structure of the two configurations on the $\mathrm{Cu}(111)$ surface is consistent with the chemical structure of $\mathrm{TiOPc}$ molecule and indicates a flat-lying adsorption configuration.

The characteristic central features of MPcs could be the contribution of molecular geometric configuration or the local density of electronic states close to the Fermi level and their convolution [21,22]. It is difficult to identify the actual molecular structures from the corresponding STM topography only according to the molecular geometry. For the planar MPc/metal system, the central metal ions appear either as a depression $(\mathrm{M}=\mathrm{Ni}, \mathrm{Cu}$ and $\mathrm{Zn})$ [23-25] or a protrusion ( $\mathrm{M}=\mathrm{Fe}, \mathrm{Co}$ and $\mathrm{Mn}$ ) [26-28], depending on the energy of $d_{Z}^{2}$ orbital with respect to the Fermi level. The non-planar MPc molecules also prefer a flat-lying adsorption configuration on metal surface mostly due to the strong interaction between the $\pi$ system of Pc skeleton and the metal substrate. The adsorption configurations could be in two opposite orientations due to their non-planarity. The observed central protrusion feature is attributed to an upward orientation, and the depression feature to a downward orientation, which is reasonable for non-planar MPcs with pure metal, such as $\mathrm{SnPc}$ [8] and $\mathrm{PbPc}$ [29]. It has been reported that such attribution is not always reliable especially for MPcs with $\mathrm{O}$ or $\mathrm{Cl}$ atom bonded to the metal ions. Barlow et al. [9] has reported an O-up configuration with a central depression and four bright lobes for VOPc adsorbed on an $\mathrm{Au}(111)$ surface. Hence, we cannot simply assign the TiOPc-pro to an O-up configuration and the TiOPc-dep to an O-down configuration. The correlation between the apparent topography and actual molecular orientation should be assigned based on detailed information obtained from both STM imaging and dipole associated work function measurement.

Non-planar polar molecules can adopt dipole up and down configurations on the substrate. The opposite dipoles can increase or decrease the local WF of the sample. We further investigated the different configurations of TiOPc molecule and the molecule-substrate interaction as well as their effects on the interface dipole by measuring the resultant LCPD using a qPlus NC-AFM. The measured frequency shift $\Delta f$ with respect to the sample bias $V(\Delta f(V)$ spectra) at a constant tip height gives a parabola due to the electric force between tip and sample. LCPD is defined as $e V_{\mathrm{CPD}}$ ( $e$ is the electron charge), which is measured by determining the peak position of the $\Delta f(V)$ curve [12]. The corresponding maximum in the $\Delta f(V)$ curve is $\Delta f^{*}$, i.e. the $\Delta f$ at the compensated $V_{\mathrm{CPD}}$. In all the $\Delta f(\mathrm{~V})$ measurements: The tip was first positioned on the center of the molecule in constant-current mode (with tuning fork oscillating); Then the feedback loop was open and the sample bias voltage ramped over the predetermined range. During the measurements of the $\Delta f(V)$, the $z$ scale was corrected by the already obtained apparent height profiles in order to keep the tip at a common height relative to the substrate.

The $\Delta f(V)$ spectra on TiOPc-pro, TiOPc-dep and $\mathrm{Cu}(111)$ surface at the constant tip height with respect to the $\mathrm{Cu}(111)$ substrate are shown in Figure 2. The LCPD shifts of TiOPcpro and TiOPc-dep molecules were $\sim 34 \pm 10 \mathrm{meV}$ and $-39 \pm$ $10 \mathrm{meV}$ with respect to the $\mathrm{Cu}(111)$ surface, respectively.
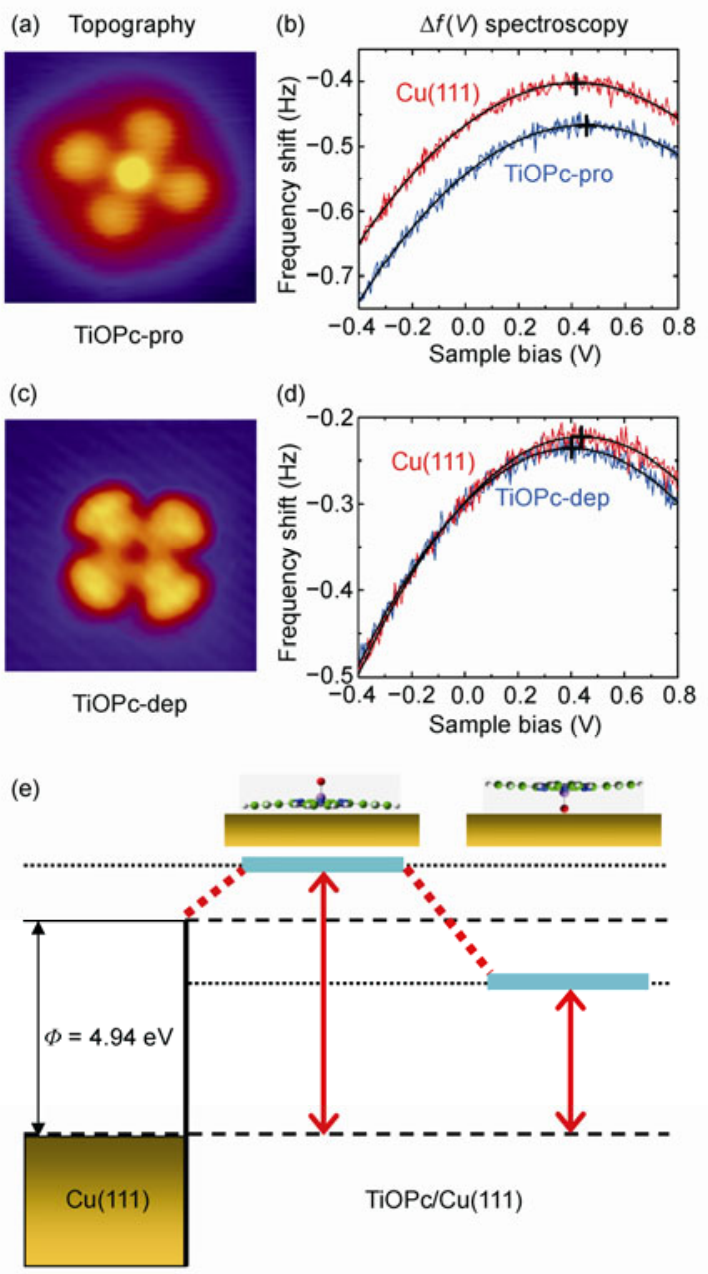

Figure 2 Close-up STM topography of (a) TiOPc-pro molecule $\left(I_{\mathrm{t}}=100\right.$ $\left.\mathrm{pA}, V_{\text {bias }}=0.5 \mathrm{~V}, 2.5 \times 2.5 \mathrm{~nm}^{2}\right)$ and (c) TiOPc-dep molecule $\left(I_{\mathrm{t}}=100 \mathrm{pA}\right.$, $V_{\text {bias }}=-1.0 \mathrm{~V}, 2.5 \times 2.5 \mathrm{~nm}^{2}$ ) on $\mathrm{Cu}(111)$ surface. Frequency shift as a function of the voltage above the center of (b) TiOPc-pro and (d) TiOPcdep molecules with respect to that of the pristine $\mathrm{Cu}(111)$ surface (oscillation amplitude $\left.A=1.0 \mathrm{~nm}, I_{\mathrm{t}}=100 \mathrm{pA}, V_{\text {bias }}=-1.0 \mathrm{~V}\right)$. Parabolic fits and corresponding parabola peaks are indicated. Blue lines in (b) and (d) represent the $\Delta f(V)$ curves on the TiOPc molecules and the red lines for the $\Delta f(V)$ curves on $\mathrm{Cu}(111)$ substrate. (e) Sketch illustrating the molecular orientation dependence of local WF shifts (red arrow) at $\mathrm{TiOPc} / \mathrm{Cu}(111)$ interface with respect to the pristine $\mathrm{Cu}(111)$ surface. 
The shifts in the LCPD can be explained by the interface dipole formed at the molecule/metal interface. In their pioneering paper, Gross et al. [13] had attributed the observed LCPD shift of $\mathrm{Au}^{-}$with respect to $\mathrm{Au}^{0}$ on $\mathrm{NaCl}(2 \mathrm{ML}) / \mathrm{Cu}(111)$ to the dipole moment directed from the vacuum to the surface induced by the negative $\mathrm{Au}^{-}$and its screening charge in the underlying substrate. In the case of TiOPc-pro/ $\mathrm{Cu}(111)$, the increase of LCPD at the TiOPc-pro molecule position is due to the interface dipole moment pointing to the sample and the sample bias has to be more positive to compensate the dipole moment. The decrease of LCPD at the TiOPc-dep molecule position indicates a surface dipole moment pointing to the vacuum, which lowers the local WF at the TiOPc-dep position and explains the negative LCPD shift with respect to that of $\mathrm{Cu}(111)$ surface. Note that the observed values of the LCPD shifts cannot be correlated to the amplitude of the dipole in a simple way because they were crucially dependent on the exact tip structure and composition. However, the observed directions in the LCPD shift can be readily assigned due to the only dependence on the adsorption configurations of TiOPc molecules.

Figure 2(e) illustrates the local WF shift depending on the adsorption configurations of TiOPc molecules. The observed LCPD shifts are in opposite direction and are consistent with the oppositely oriented permanent dipole induced by the TiOPc-pro and TiOPc-dep molecules, respectively. Similar to the $\mathrm{CuPc} / \mathrm{Cu}(111)$ system [18], TiOPc molecules tend to cover the steps first, which indicates a weak interaction between the molecules and the substrate. We believe that the dipole induced by charge transfer between TiOPc molecules and the $\mathrm{Cu}(111)$ surface may play a minor role comparing to the large permanent dipole of the TiOPc molecules. Hence, the different molecular features, TiOPc-pro and TiOPc-dep, observed in STM images are attributed to O-up and O-down configurations, respectively. This is contrast to the results of vanadyl naphthalocyanine grown on $\mathrm{Au}(111)$ surface reported by Terentjevs et al. [4]. They report a large contribution of "push-back" effect and a minor role of permanent molecular dipole in the work function reduction of the interface. The distinct origin of interface dipole may be due to the different interaction between the molecules and substrates.

It is well known that the charge transfer between the molecule and the substrate strongly influences the orientation and magnitude of interface dipole moment, leading to a strong dependence on the properties of the substrate. In order to study the effect of different substrates on the WF modification and to extend the measurements of LCPD to broader systems, we performed the $\Delta f(V)$-measurements on the $\mathrm{TiOPc} / \mathrm{Cu}(100)$ system using the same method above.

The TiOPc molecules on $\mathrm{Cu}(100)$ surface still prefer the flat-lying adsorption configuration. The two kinds of molecular central characteristics, protrusion and depression, can still be observed, as shown in Figure 3(a) and (c). The



TiOPc-pro

(c)

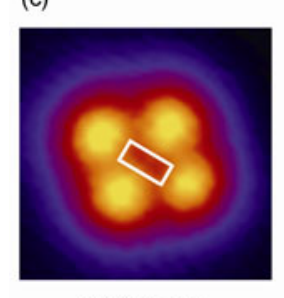

TiOPc-dep

(e)

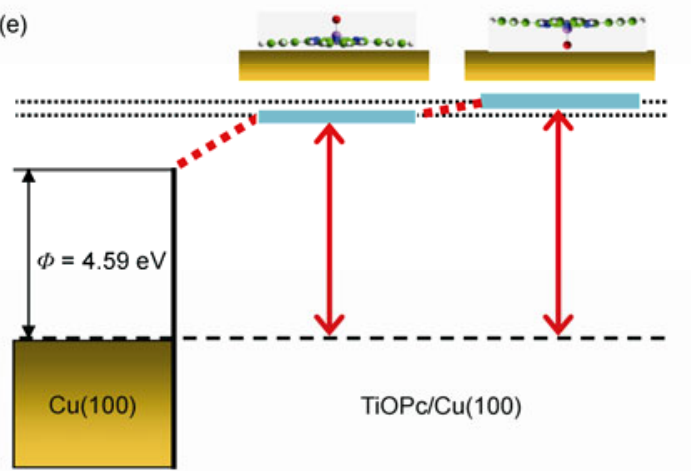

Figure 3 Close-up STM topography of (a) TiOPc-pro molecule ( $I_{\mathrm{t}}=100$ $\left.\mathrm{pA}, V_{\text {bias }}=1.0 \mathrm{~V}, 2.5 \times 2.5 \mathrm{~nm}^{2}\right)$ and (c) TiOPc-dep molecule $\left(I_{\mathrm{t}}=100 \mathrm{pA}\right.$, $\left.V_{\text {bias }}=0.5 \mathrm{~V}, 2.5 \times 2.5 \mathrm{~nm}^{2}\right)$ on $\mathrm{Cu}(100)$ surface. The rectangle in (c) indicates the two-fold symmetry of the TiOPc-dep molecule. Frequency shift as a function of the voltage above the center of (b) TiOPc-pro and (d) TiOPc-dep molecule with respect to that of the pristine $\mathrm{Cu}(100)$ surface (oscillation amplitude $A=1.35 \mathrm{~nm}, I_{\mathrm{t}}=20 \mathrm{pA}, V_{\text {bias }}=1.0 \mathrm{~V}$ ). Parabolic fits and corresponding parabola peaks are indicated. Blue lines in (b) and (d) represent the $\Delta f(V)$ curves on the TiOPc molecules and the red lines for the $\Delta f(V)$ curves on $\mathrm{Cu}(100)$ substrate. (e) Sketch illustrating the molecular orientation dependence of local WF shifts (red arrow) at $\mathrm{TiOPc} / \mathrm{Cu}(100)$ interface with respect to the pristine $\mathrm{Cu}(100)$ surface.

corresponding LCPD shift between TiOPc-pro and $\mathrm{Cu}(100)$ surface was $\sim 17 \pm 2 \mathrm{meV}$, as shown in Figure 3(b). The observed LCPD shift is in the same direction as that induced by the permanent dipole of TiOPc-pro molecule. The corresponding LCPD shift between TiOPc-dep and $\mathrm{Cu}(100)$ surface was 26 $2 \mathrm{meV}$ (Figure 3(d)), which is in the opposite direction as that induced by the permanent dipole of the TiOPc-dep molecule. The WF shift depending on the molecular configurations of TiOPc molecules are illustrated in Figure 3(e). The local WF above the TiOPc-pro and TiOPc-dep molecules shifts in the same direction.

It is interesting to note that the interface dipole moment above TiOPc-dep on $\mathrm{Cu}(100)$ points to the substrate and opposite to the permanent dipole moment of TiOPc-dep 
molecule. Generally, the LCPD at a metal/organic interface can be modified through three mechanisms: (i) electron density "push-back" effect at the metal surface, (ii) the permanent dipole of the organic molecule and (iii) the "bonding" dipole induced by a partial charge transfer between the organic molecule and the metal. Considering that the "push-back" effect and the permanent dipole moment pointing to the vacuum will reduce the local WF, the interface dipole moment above TiOPc-dep on $\mathrm{Cu}(100)$ should be resulted from the strong charge transfer from the $\mathrm{Cu}(100)$ surface. This is in analogy to the case of ClAlPc adsorbed on $\mathrm{Cu}(111)$ with $\mathrm{Cl}$-down configuration reported by Niu et al. [5], in which a significant charge was donated from the $\mathrm{Cu}(111)$ surface to Cl-down adsorbed ClAlPc molecule and this charge transfer led to a symmetry reduction for Cl-down adsorbed ClAlPc from $C_{4 v}$ to $C_{2 v}$. It should be noted that the linear central depression of the TiOPc-dep molecule on the $\mathrm{Cu}(100)$ surface (Figure 3(c)) also indicates a 2-fold symmetric feature of the TiOPc-dep/ $\mathrm{Cu}(100)$ system. The observed symmetry reduction could be resulted from the incommensurability of the 4-fold symmetric phthalocyanine and 6-fold symmetric metal surface [30] or the charge redistribution within the Pc ring because of the charge transfer between molecule and the substrate [5,31]. Considering the weak coupling of Pc ring with the substrate for the O-down configuration and the unobservable symmetry reduction for the TiOPc-pro molecule, a stronger molecule-substrate interaction can be suggested for the TiOPc-dep/Cu(100) system and the charge transfer between $\mathrm{O}$ atom and the copper surface leads to the charge redistribution within the Pc ring. Hence, it is the significant charge redistribution that dominates the permanent dipole moment of TiOPc-dep molecule and results in the interface dipole moment pointing to the substrate. Comparing to the $\mathrm{Cu}(100)$ surface, the $\mathrm{Cu}(111)$ surface has larger WF [32] and is inerter in reactivity. These differences might lead to weaker charge transfer between the TiOPc-dep molecule and $\mathrm{Cu}(111)$ surface and weaker "bonding" dipole. Therefore, the permanent dipole of TiOPc-dep molecule dominates the interface dipole of the TiOPc-dep molecules on $\mathrm{Cu}(111)$ surface and leads to the same orientation of the permanent dipole and the interface dipole. The adsorption of MPcs on $\mathrm{Cu}(111)$ and $\mathrm{Cu}(100)$, respectively, can provide a valuable contribution to the understanding of molecule-substrate interactions and shed light on tailoring interfacial electronic properties and controlling local physical properties via polar molecule adsorption.

\section{Conclusion}

In summary, we presented a combined STM and NC-AFM investigation on the orientation-specific electric dipole moments of polar TiOPc molecules on $\mathrm{Cu}(111)$ and $\mathrm{Cu}(100)$ surfaces. For TiOPc adsorbed on $\mathrm{Cu}(111)$ surface, the ob- served interface dipole moments are in the same direction as the permanent dipoles of the TiOPc-pro and TiOPc-dep molecules. We correlate the actual molecular structures with the corresponding STM topographies by combining the sub-molecular resolved STM images and LCPD measurements. However, the interface dipole moment above TiOPc-dep on $\mathrm{Cu}(100)$ is observed to be opposite to the permanent dipole moment of TiOPc-dep, which could be attributed to the significant charge transfer between $\mathrm{Ti}_{-}$ OPc-dep and $\mathrm{Cu}(100)$ surface. The LCPD shift due to the adsorbed non-planar molecule is the contribution of the permanent dipole of the molecule, the molecular adsorption configurations, and the charge transfer between the substrate and the molecule. The results shown here could further the understanding of the microscopic structural and interfacial electronic properties of the molecule as it adsorbed on different metallic substrates and foster investigations on molecular electronics and electronic properties of metal/organic interface.

This work was supported by the National Basic Research Program of China (2012CB933001) and the National Natural Science Foundation of China $(21173058,21203038)$.

1 Ishii H, Sugiyama K, Ito E, et al. Energy level alignment and interfacial electronic structures at organic/metal and organic/organic interfaces. Adv Mater, 1999, 11: 605-625

2 Heimel G, Salzmann I, Duhm S, et al. Design of organic semiconductors from molecular electrostatics. Chem Mater, 2010, 23: 359-377

3 Gerlach A, Hosokai T, Duhm S, et al. Orientational ordering of nonplanar phthalocyanines on $\mathrm{Cu}(111)$ : Strength and orientation of the electric dipole moment. Phys Rev Lett, 2011, 106: 156102

4 Terentjevs A, Steele M P, Blumenfeld M L, et al. Interfacial electronic structure of the dipolar vanadyl naphthalocyanine on $\mathrm{Au}(111)$ : "Push-back" vs dipolar effects. J Phys Chem C, 2011, 115: 21128-21138

5 Niu T, Zhou M, Zhang J, et al. Dipole orientation dependent symmetry reduction of chloroaluminum phthalocyanine on $\mathrm{Cu}(111)$. J Phys Chem C, 2013, 117: 1013-1019

6 Heimel G, Romaner L, Zojer E, et al. The interface energetics of self-assembled monolayers on metals. Acc Chem Res, 2008, 41: 721-729

7 Martinez-Diaz M V, de la Torre G, Torres T. Lighting porphyrins and phthalocyanines for molecular photovoltaics. Chem Commun, 2010, 46: 7090-7108

8 Wang Y, Kröger J, Berndt R, et al. Structural and electronic properties of ultrathin tin-phthalocyanine films on $\operatorname{Ag}(111)$ at the single-molecule level. Angew Chem Int Ed, 2009, 48: 1261-1265

9 Barlow D E, Hipps K W. A scanning tunneling microscopy and spectroscopy study of vanadyl phthalocyanine on Au(111): The effect of oxygen binding and orbital mediated tunneling on the apparent corrugation. J Phys Chem B, 2000, 104: 5993-6000

10 Duncan D A, Unterberger W, Hogan K A, et al. A photoelectron diffraction investigation of vanadyl phthalocyanine on $\mathrm{Au}(111)$. Surf Sci, 2010, 604: 47-53

11 Glatzel T. Measuring atomic-scale variations of the electrostatic force. In: Sadewasser S, Glatzel T, eds. Kelvin Probe Force Microscopy. Berlin: Springer, 2012. 289-327

12 Melitz W, Shen J, Kummel A C, et al. Kelvin probe force microscopy and its application. Surf Sci Rep, 2011, 66: 1-27

13 Gross L, Mohn F, Liljeroth P, et al. Measuring the charge state of an 
adatom with noncontact atomic force microscopy. Science, 2009, 324: 1428-1431

14 Mohn F, Gross L, Moll N, et al. Imaging the charge distribution within a single molecule. Nat Nanotechnol, 2012, 7: 227-231

15 König T, Simon G H, Rust H P, et al. Measuring the charge state of point defects on $\mathrm{MgO} / \mathrm{Ag}(001)$. J Am Chem Soc, 2009, 131: 1754417545

16 Giessibl F J. Atomic resolution on $\mathrm{Si}(111)-(7 \times 7)$ by noncontact atomic force microscopy with a force sensor based on a quartz tuning fork. Appl Phys Lett, 2000, 76: 1470-1472

17 Fukagawa H, Yamane H, Kera S, et al. Experimental estimation of the electric dipole moment and polarizability of titanyl phthalocyanine using ultraviolet photoelectron spectroscopy. Phys Rev B, 2006, 73: 041302

18 Chang S H, Kuck S, Brede J, et al. Symmetry reduction of metal phthalocyanines on metals. Phys Rev B, 2008, 78: 233409

19 Wei Y, Robey S W, Reutt-Robey J E. Flux-selected titanyl phthalocyanine monolayer architecture on $\mathrm{Ag}(111)$. J Phys Chem C, 2008, 112: 18537-18542

20 Wei Y, Robey S W, Reutt-Robey J E. TiOPc molecular dislocation networks as nanotemplates for C60 cluster arrays. J Am Chem Soc, 2009, 131: 12026-12027

21 Lu X, Hipps K W, Wang X D, et al. Scanning tunneling microscopy of metal phthalocyanines: d7 and d9 Cases. J Am Chem Soc, 1996, 118: 7197-7202

22 Lu X, Hipps K W. Scanning tunneling microscopy of metal phthalocyanines: d6 and d8 cases. J Phys Chem B, 1997, 101: 5391-5396
23 Koudia M, Abel M, Maurel C, et al. Influence of chlorine substitution on the self-assembly of zinc phthalocyanine. J Phys Chem B, 2006, 110: 10058-10062

24 Mugarza A, Krull C, Robles R, et al. Spin coupling and relaxation inside molecule-metal contacts. Nat Commun, 2011, 2: 490

25 Uhlmann C, Swart I, Repp J. Controlling the orbital sequence in individual Cu-phthalocyanine molecules. Nano Lett, 2013, 13: 777-780

26 Cheng Z H, Gao L, Deng Z T, et al. Adsorption behavior of iron phthalocyanine on $\mathrm{Au}(111)$ surface at submonolayer coverage. J Phys Chem C, 2007, 111: 9240-9244

27 Fu Y S, Ji S H, Chen X, et al. Manipulating the kondo resonance through quantum size effects. Phys Rev Lett, 2007, 99: 256601

28 Heinrich B W, Iacovita C, Brumme T, et al. Direct observation of the tunneling channels of a chemisorbed molecule. J Phys Chem Lett, 2010, 1: 1517-1523

29 Baran J D, Larsson J A, Woolley R A J, et al. Theoretical and experimental comparison of $\mathrm{SnPc}, \mathrm{PbPc}$, and $\mathrm{CoPc}$ adsorption on Ag(111). Phys Rev B, 2010, 81: 075413

30 Cuadrado R, Cerda J I, Wang Y, et al. CoPc adsorption on $\mathrm{Cu}(111)$ : Origin of the C4 to C2 symmetry reduction. J Chem Phys, 2010, 133: 154701

31 Mugarza A, Lorente N, Ordejón P, et al. orbital specific chirality and homochiral self-assembly of achiral molecules induced by charge transfer and spontaneous symmetry breaking. Phys Rev Lett, 2010, 105: 115702

32 Skriver H L, Rosengaard N M. Surface energy and work function of elemental metals. Phys Rev B, 1992, 46: 7157-7168

Open Access This article is distributed under the terms of the Creative Commons Attribution License which permits any use, distribution, and reproduction in any medium, provided the original author(s) and source are credited. 